\title{
Seguridad de la anestesia neuroaxial en mujeres con embarazo gemelar y síndrome de transfusión feto- fetal, sometidas a fotocoagulación láser. Estudio de cohorte retrospectiva
}

\section{Safety of neuraxial anesthesia in patients twin pregnancy and twin-to-twin transfusion syndrome taken to laser photocoagullation. Retrospective cohort study}

Luis Felipe Laverde-Martínez, MD ${ }^{1,3}$; Laura Marcela Zamudio-Castilla, MD ${ }^{1}$; Akemi Arango-Sakamoto ${ }^{2}$; Natalia Satizábal-Padridin, $M^{2}$; Leidy Johanna López-Erazo, MD $^{3}$; Einar Sten Billefals-Vallejo, MD³; Yuliana Angélica Orozco-Peláez, $\mathrm{MD}^{3}$

Recibido: 30 de diciembre de 2020/Aceptado: 2 de agosto de 2021

\section{RESUMEN}

Objetivo: describir las características clínicas y la frecuencia de complicaciones maternas, fetales y neonatales, según técnica de anestesia neuroaxial (AN) en mujeres con síndrome de transfusión feto-fetal (STFF) tratadas con fotocoagulación láser (FL).

Materiales y métodos: estudio de cohorte retrospectiva descriptivo. Se incluyeron gestantes con STFF tratadas con FL y AN en la Fundación Valle del Lili, Cali (Colombia) entre 2013-2017. Se excluyeron pacientes con STFF estadio-V de Quintero. Se usó estadística descriptiva. El protocolo fue aprobado por el Comité de Ética de la institución. Resultados: 32 participantes cumplieron con los criterios de inclusión y de exclusión. La población estuvo constituida por mujeres jóvenes, multíparas.

* Correspondencia: Luis Felipe Laverde-Martínez, Fundación Valle del Lili, carrera 98 No. 18-49, Cali (Colombia). Teléfono: (57) (2) 3319090, ext. 4022. luis. laverde@fvl.org.co

1. Universidad Icesi, Facultad de Ciencias de la Salud, Departamento de Ciencias Clínicas, Cali (Colombia).

2. Fundación Valle del Lili, Centro de Investigaciones Clínicas, Cali (Colombia)

3. Fundación Valle del Lili, Departamento de Anestesiología, Cali (Colombia)
En el 87,5\% de los casos se realizó intervención de urgencia. El 43,7\% presentaba el estadio-III de Quintero y en el 56,2\% de las gestantes se utilizó anestesia epidural. Las variables hemodinámicas maternas exhibieron un comportamiento similar, acorde al momento de la cirugía y la técnica neuoraxial implementada. El 65,6\% de las gestantes presentó hipotensión sostenida y el 9,3\% desarrolló edema pulmonar. El 65,6\% de las pacientes experimentó parto pretérmino y el 18,7\% ruptura prematura de membranas. Se registraron 14 muertes fetales y cinco neonatales. No se registraron casos de mortalidad materna.

Conclusiones: en pacientes con STFF que requieren FL, el uso de la anestesia epidural, espinal o combinada probablemente se asocia con un comportamiento similar al de las variables hemodinámicas maternas, durante los momentos de la cirugía. Los profesionales que brindan atención a estas gestantes deben estar alerta ante la frecuente aparición de complicaciones maternas, fetales y neonatales. Se requieren estudios prospectivos que evalúen la seguridad y la efectividad de las diferentes 
técnicas de anestesia neuroaxial en pacientes con STFF.

Palabras clave: embarazo gemelar, transfusión feto-fetal, anestesia, anestesia epidural, anestesia raquídea, fotocoagulación láser, fetoscopia.

\section{ABSTRACT}

Objective: To describe the clinical characteristics and the frequency of maternal, fetal and neonatal complications in accordance with the neuraxial anesthesia (NA) technique in women with twin-totwin transfusion syndrome (TTTS) treated with laser photocoagulation.

Materials and Methods: Descriptive retrospective cohort study of pregnant patients with TTTS treated with laser photocoagulation under NA at Fundación Valle del Lili, Cali (Colombia), between 2013-2017. Patients with Quintero stage VTTTS were excluded. The protocol was approved by the institutional ethics committee.

Results: Of the participants, 32 met the inclusion and exclusion criteria. The study population consisted of young, multiparous women. Urgent interventions were performed in $87.5 \%$ of cases; $43.7 \%$ were Quintero stage III and epidural anesthesia was used in 56.2\% of the women. Maternal hemodynamic variables were similar, in accordance with the timing of surgery and the neuraxial technique used. Sustained hypotension occurred in $65.6 \%$ of the pregnant women and 9.3\% developed pulmonary edema. Pre-term delivery occurred in $65.6 \%$ of the patients and $18.7 \%$ had premature rupture of membranes. There were 14 fetal demises and five neonatal deaths. There were no cases of maternal mortality.

Conclusions: In patients with TTTS requiring laser photocoagulation, the use of epidural, spinal or combined anesthesia is likely associated with similar maternal hemodynamic variables at the time of surgery. Practitioners providing care to these pregnant women must be aware of the frequent occurrence of maternal, fetal and neonatal complications. Prospective studies to assess the safety and effectiveness of the different neuraxial anesthesia techniques in patients with TTTS are required.
Keywords: Monozygotic twins, fetofetal transfusion, anesthesia, epidural anesthesia, spinal anesthesia, general anesthesia.

\section{INTRODUCCIÓN}

El síndrome de transfusión feto-fetal (STFF) es una condición específica de los embarazos gemelares monocoriales (1), que se caracteriza por la presencia de anastomosis arteriovenosas profundas en el lecho capilar de los cotiledones $(2,3)$, lo que lleva al desarrollo de flujo unidireccional no balanceado entre los fetos $(4,5)$, con la subsecuente hipovolemia en el gemelo donante e hipervolemia en el receptor (6). Aunque la frecuencia exacta de STFF se desconoce, se estima que compromete del 10 al $30 \%$ de los embarazos monocoriales monocigóticos (2), gestaciones en las que se incrementa la frecuencia de resultado perinatal adverso $(5,6)$ dada su estrecha asociación con la presencia de muerte o alteraciones del crecimiento fetal (80\% de los fetos comprometidos), en especial, si no se brinda tratamiento oportuno (7).

De acuerdo con el estadio de la enfermedad y su progresión, la secuencia de mecanismos fisiopatológicos $(8,9)$ se correlaciona con los hallazgos ecográficos y la categorización de la enfermedad en estadios de I a V según la clasificación propuesta por Quintero (10). De esta forma, el espectro de la enfermedad abarca desde un primer estadio que corresponde a la presencia de polihidramnios en el gemelo receptor (bolsillo vertical $>8 \mathrm{~cm}$ ), acompañado de oligohidramnios en el feto donante (bolsillo vertical $<2 \mathrm{~cm}$ ), ausencia de vejiga visible en el feto donante (II), presencia de doppler anormal (III), desarrollo de hidrops fetal (IV), y culminando con la muerte de uno o ambos gemelos (estadio V) (8).

El manejo del STFF varía en consonancia con el estadio clínico de la enfermedad, el primer estadio suele ser objeto de manejo expectante (1) sopesando el riesgo de progresión cercano al $15 \%$ (4). Por su parte, para los estadios II en adelante no existen alternativas farmacológicas seguras y efectivas (11), lo que deja en manos de los procedimientos fetales invasivos (v. g. amniorreducción, feticidio selectivo, 
fotocoagulación láser y septostomia) el pronóstico de este tipo de gestaciones (11). No obstante, la evidencia creciente apunta al uso preferente de la fotocoagulación láser, dada su asociación con mayor sobrevida y con mejores puntajes en la escala de neurodesarrollo a los seis años de vida, cuando se compara frente a otras alternativas (Razón de Riesgos [RR] 1.57; Intervalo de Confianza [IC] $95 \%$ : 1,05-2,34) (12).

Pero los vacíos del conocimiento no solo pasan por la elección apropiada de la intervención fetal, también abarcan la selección de la técnica anestésica a la hora de realizar la cirugía fetal (13). Para el manejo de las gestantes afectadas con STFF, se puede implementar el uso de anestesia general o de técnicas neuroaxiales, solas o combinadas (14-18). La decisión de la técnica tendrá como fundamento el abordaje quirúrgico planeado (13), la probabilidad de convertir a cirugía abierta (17), la localización de la placenta y del feto $(2,14)$, el deseo de inmovilidad fetal, el grado de analgesia y sedación materno-fetal deseado y, finalmente, la experiencia clínica (2).

Si bien el uso de anestesia general puede asegurar mejores niveles de sedación y analgesia, esta técnica se ha asociado con un mayor consumo de oxígeno y una incidencia más alta de hipotensión materna e hipoxia fetal secundaria (19-20). Al comparar la anestesia general frente a otros abordajes, un estudio observacional evidenció que esta presenta mayor fluctuación de la frecuencia cardiaca y tensión arterial materna, lo que conlleva una mayor tasa de complicaciones (21). Por su parte, las técnicas neuroaxiales (comprenden la aproximación espinal, epidural o combinada) (13) presentan como principal ventaja: un menor riesgo teórico de regurgitación gástrica (14), la menor exposición fetal a los agentes anestésicos (22), junto a un efecto limitado sobre la hemodinamia fetal y el flujo uteroplacentario si se mantiene la presión arterial media materna (18). Como principales desventajas para este abordaje se encuentran la falta de anestesia o analgesia fetal, el riesgo potencial de falla en el bloqueo y la inyección intravascular inadvertida del anestésico (10).
En la actualidad no existen estudios con suficiente poder o rigor metodológico que analicen la seguridad y la efectividad de las diferentes alternativas de analgesia para las pacientes con STFF que requieran fetoscopia con fotocoagulación láser $(15,23)$. Los vacíos del conocimiento y controversias existentes, explican la alta variabilidad clínica en el manejo anestésico y dejan la toma de decisiones en la experiencia y preferencia del profesional a cargo $(2,12,14,22,24)$. Con esto en mente, el objetivo de este estudio es describir las características clínicas y la frecuencia de complicaciones maternas, fetales y neonatales, acordes a la técnica de anestesia neuroaxial, en mujeres con diagnóstico de STFF, tratadas con fotocoagulación láser.

\section{MATERIALES Y MÉTODOS}

Diseño y población. Estudio de cohorte retrospectiva descriptivo. Se incluyeron todas las gestantes con diagnóstico de síndrome de transfusión feto-fetal (STFF), tratadas con fetoscopia y fotocoagulación láser con anestesia neuroaxial, entre enero de 2013 y diciembre de 2017 en la Fundación Valle del Lili, entidad de naturaleza privada, centro de referencia de alto nivel de complejidad, ubicada en la ciudad de Cali (Colombia), que atiende población adscrita al régimen subsidiado y contributivo del Sistema General de Seguridad Social en Salud (SGSSS). Se excluyeron pacientes con STFF estadio V de Quintero (muerte de uno o ambos fetos) (10), anomalía fetal mayor conocida o sospecha de aneuploidía. Se implementó un muestreo consecutivo por conveniencia a partir del universo de pacientes atendidas en la institución participante durante el periodo de estudio. Tamaño de muestra: no se realizó cálculo de tamaño de muestra.

Procedimiento. Las pacientes fueron identificadas con el apoyo del departamento de estadística. Se solicitaron todos los registros identificados con el código CIE-10 O430 (25) que corresponde al diagnóstico "síndrome de transfusión placentaria"; se realizó el cribado inicial de historias clínicas utilizando como filtro el código CUPS 753701 que identifica el pro- 
cedimiento "fetoscopia" (26). Una vez surtido este paso, dos investigadores revisaron de forma independiente las historias recuperadas y, aplicando los criterios de inclusión y exclusión, seleccionaron la población objeto de estudio. Posteriormente, previo diseño de un formulario de recolección de datos, dos autores extrajeron la información de interés utilizando el programa BD Clinic, con prueba piloto, para luego verificar la calidad de los datos mediante revisión pareada del 10\% de las historias clínicas incluidas (selección por muestreo aleatorio). No se encontraron discrepancias.

Un equipo de 14 anestesiólogos proporcionó la técnica anestésica. Para todas las punciones se realizó asepsia y antisepsia en la región lumbar e infiltración de la piel y el tejido celular subcutáneo con anestésico local sin epinefrina (20). Para la anestesia epidural se realizó punción lumbar y se ubicó el espacio peridural con técnica de pérdida de resistencia al aire (20). Al comprobar aspiración negativa se realizó prueba con bupivacaína con o sin opioide y se insertó catéter en el espacio peridural con aguja 16-18 G (20). En la técnica espinal, se insertó aguja punta de lápiz calibre 25-27 G en el espacio intervertebral y al evidenciar la salida de líquido cefalorraquídeo se inyectó anestésico local (20). En lo que respecta a la intervención, la fotocoagulación fue realizada por un especialista en medicina materno-fetal con entrenamiento en terapia fetal, con posterior cuidado intra hospitalario a cargo de los servicios de obstetricia y ginecología, y medicina materno-fetal.

Variables medidas. Las variables relevantes para el estudio fueron: edad materna, índice de masa corporal (IMC), número de gestaciones previas, presencia de comorbilidades maternas (v. g. patología de origen endocrino, trastorno hipertensivo o enfermedad hematológica), edad gestacional al momento del procedimiento, estadio de Quintero, tipo de cirugía (electiva o urgente), técnica de anestesia neuroaxial (epidural, espinal o combinada), duración del procedimiento, signos vitales maternos al momento de la cirugía (después de la inducción, antes de la incisión y al fin del procedimiento), complicaciones maternas o fetales asociadas a la intervención (v. g. incidencia de parto pretérmino, ruptura prematura de membranas, edema pulmonar, muerte materna o fetal, lesión de víscera hueca) o relacionadas con la anestesia (v. g. hipotensión sostenida, hipoxemia materna), tipo de analgesia en el posoperatorio y sobrevida del neonato al tercer mes.

Análisis estadístico. Se utilizó el paquete estadístico STATA $^{\circledR} 14$. Se realizó estadística descriptiva para las variables clínicas y sociodemográficas. Las variables cualitativas se presentan como frecuencias absolutas y relativas, y para las cuantitativas se emplearon medidas de tendencia central y dispersión según la distribución de los datos analizados, la cual fue evaluada con la prueba de Shapiro-Wilk. Se utilizó promedio y desviación estándar cuando la distribución cumplió criterios de normalidad, y, en caso contrario, se aplicó mediana y rango intercuartílico.

Aspectos éticos. El protocolo para este estudio fue aprobado por el Comité de Ética en Investigación Biomédica de la Fundación Valle del Lili participante (número de protocolo 1211). Se garantizó la confidencialidad y el anonimato de la información.

\section{RESULTADOS}

Se identificaron 40 pacientes con diagnóstico de STFF, de las cuales 34 fueron llevadas a fetoscopia. Se excluyó una paciente por diagnóstico de STFF estadio V de Quintero, y una gestante que recibió anestesia total intravenosa (ATI). De esta forma, se analizó el resultado perinatal en 32 gestantes (64 fetos) que acudieron a la institución participante durante el periodo de estudio (Figura 1). No se pudo recolectar información en 4 gestantes ( 8 fetos), por lo cual la mortalidad se calculó con 28 maternas (56 fetos). La población estuvo constituida preponderantemente por mujeres jóvenes, multíparas, con sobrepeso, sin patología de base, quienes cursaban la segunda mitad de la gestación (Tabla 1).

En el 87,5\% de los casos la intervención se realizó de emergencia ante la evidencia de compromiso fetal; predominó el estadio III de Quintero (43,75 \%), seguido por los estadios II (34,38\%), IV (12,5\%) y I 
Figura 1.

Diagrama de flujo que muestra la selección de las participantes en el estudio.

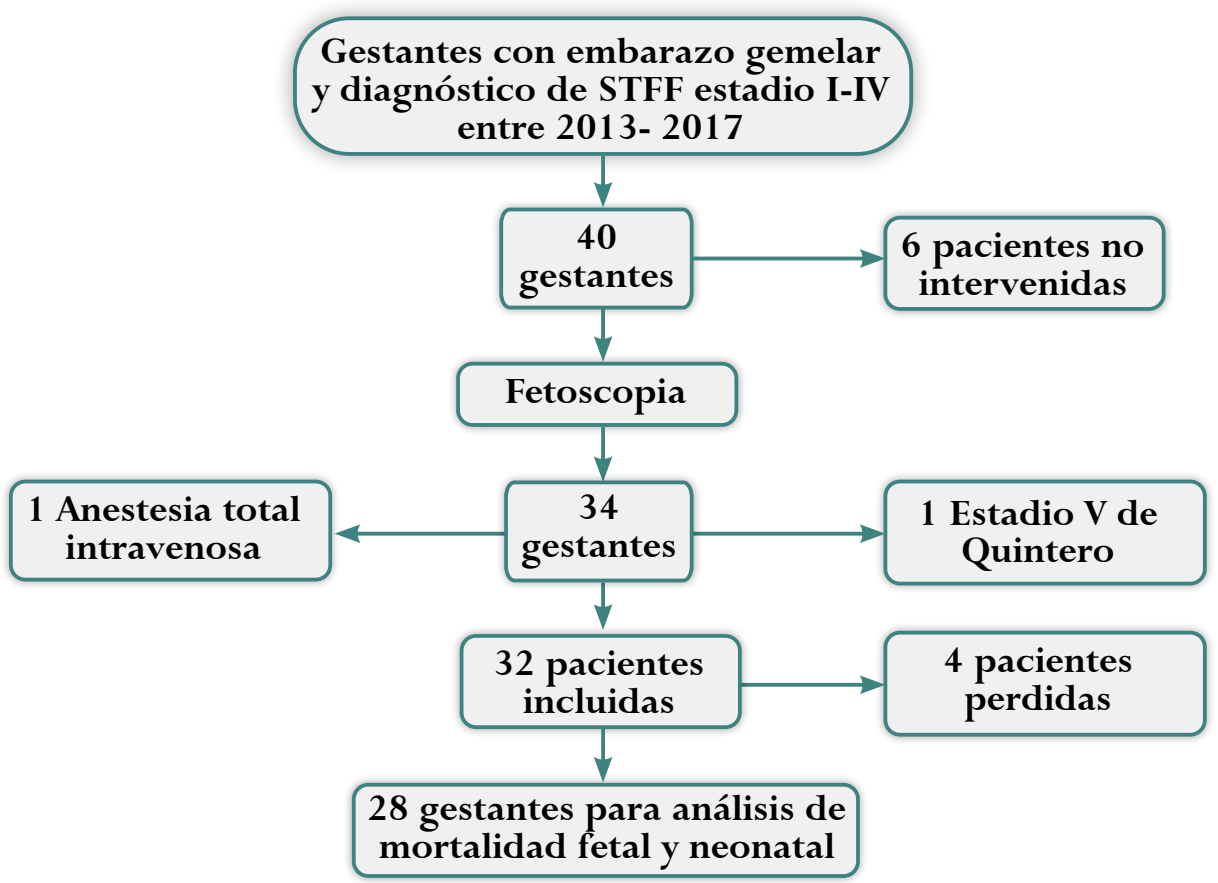

(9,38 \%); la mediana de duración del procedimiento fue de 57,5 min. (rango intercuartílico (RIQ] 55-65) para el grupo de anestesia epidural; 57,5 min. (RIQ
50-65) para las pacientes con anestesia espinal y, finalmente, 55 min. (RIQ 45-60) para las participantes con anestesia combinada.

\begin{tabular}{|c|c|}
\hline \multicolumn{2}{|c|}{$\begin{array}{c}\text { Tabla } 1 . \\
\text { Características demográficas y clínicas de las participantes. }\end{array}$} \\
\hline Variables & $\mathbf{N}=32$ \\
\hline Edad (años), Prom $\pm \mathrm{DE}$ & $27,4 \pm 6,3$ \\
\hline Índice de masa corporal, Prom $\pm \mathrm{DE}$ & $26,4 \pm 4.5$ \\
\hline Edad gestacional al momento del procedimiento, Prom \pm DE & $21,4 \pm 2,4$ \\
\hline \multicolumn{2}{|l|}{ Gestaciones, n (\%) } \\
\hline Una & $11(34,4)$ \\
\hline Dos o más & $21(65,6)$ \\
\hline \multicolumn{2}{|l|}{ Prioridad del procedimiento, n (\%) } \\
\hline Electivo & $4(12,5)$ \\
\hline Urgente & $28(87,5)$ \\
\hline \multicolumn{2}{|l|}{ Comorbilidad materna, n (\%) } \\
\hline Patología origen endocrino & $4(11,8)$ \\
\hline Trastorno hipertensivo & $1(3,12)$ \\
\hline Enfermedad hematológica & $3(9,36)$ \\
\hline Otros & $5(15,6)$ \\
\hline Antecedentes quirúrgicos obstétricos, n (\%) & $11(32,4)$ \\
\hline
\end{tabular}

Prom: promedio

DE: desviación estándar. 
Respecto a la técnica anestésica predominó el uso de anestesia epidural (56,25\%), seguido por la anestesia combinada $(25,0 \%)$ y la técnica espinal $(18,72 \%)$. Para el mantenimiento de la anestesia se utilizó en 29 pacientes remifentanil $(90,62 \%)$, fentanil o propofol en una paciente respectivamente (3,12\%). En 19 participantes $(59,38 \%)$ se requirió el uso de vasopresores así, en 13 pacientes norepinefrina (40,62\%) y en 4 etilefrina (12,5\%), y en el 4,81\% de las pacientes se requirió soporte con líquidos endovenosos. En el posoperatorio, el medicamento más frecuentemente utilizado para el control analgésico fue el acetaminofén oral o endovenoso en el 59,38\% de los casos y solo el $25 \%$ de las pacientes requirió manejo de rescate con opioides (Tabla 2).

\begin{tabular}{|l|c|}
$\begin{array}{c}\text { Tabla } 2 . \\
\text { Descripción de los aspectos relacionados con el cuidado anestésico } \\
\text { en la población participante. }\end{array}$ \\
\hline \multicolumn{1}{|c|}{ Variables } \\
\hline Técnica neuroaxial, n (\%)
\end{tabular}




\begin{tabular}{|c|c|}
\hline Dosis anestésico local, n (\%) & $\mathrm{n}=24$ \\
\hline Técnica espinal* & $\mathrm{n}=6$ \\
\hline$\leq 7,5 \mathrm{mg}$ & $4(66,7)$ \\
\hline$>7,5 \mathrm{mg}$ & $2(33,3)$ \\
\hline Técnica epidural $\ddagger$ & $\mathrm{n}=18$ \\
\hline$\leq 15 \mathrm{~cm}$ & $14(77,8)$ \\
\hline$>15 \mathrm{~cm}$ & $4(22,2)$ \\
\hline Técnica combinada & $\mathrm{n}=8$ \\
\hline$\leq 7,5 \mathrm{mg}$ & $4(50)$ \\
\hline$>8 \mathrm{mg}$ & $4(50)$ \\
\hline Uso de remifentanil intraoperatorio, $n(\%)$ & $\mathrm{n}=17$ \\
\hline Concentración $\mathrm{ICO} †$ & $\mathrm{n}=17$ \\
\hline$<1 \mathrm{ng} / \mathrm{ml}$ & $1(3,12)$ \\
\hline $1-3 \mathrm{ng} / \mathrm{ml}$ & $15(46,88)$ \\
\hline$>3 \mathrm{ng} / \mathrm{ml}$ & $1(3,12)$ \\
\hline Infusión & $\mathrm{n}=14$ \\
\hline$<0,05 \mathrm{mcg} / \mathrm{kg} / \mathrm{min}$ & $3(9,37)$ \\
\hline $0,05-01 \mathrm{mcg} / \mathrm{kg} / \mathrm{min}$ & $11(34,37)$ \\
\hline Uso de norepinefrina intraoperatoria, n (\%) & $\mathrm{n}=13$ \\
\hline Bolos de $8 \mathrm{mcg}$ & $6(46,15)$ \\
\hline Infusión $<0,1 \mathrm{mcg} / \mathrm{kg} / \mathrm{min}$ & $4(30,77)$ \\
\hline Infusión $>0,1 \mathrm{mcg} / \mathrm{kg} / \mathrm{min}$ & $3(23,08)$ \\
\hline Uso de analgésicos posoperatorios, n (\%) & $\mathrm{n}=32$ \\
\hline Acetaminofén oral/endovenoso & $19(59,38)$ \\
\hline Dipirona endovenosa & $15(46,88)$ \\
\hline Analgesia epidural & $12(37,50)$ \\
\hline Opioides endovenosos & $8(25,00)$ \\
\hline Butilbromuro de hioscina oral & $9(28,12)$ \\
\hline $\begin{array}{l}\text { Infusión controlada por objetivo } \\
\text { Bupivacaína pesada 0,5\% } \\
\text { Mezcla con bupivacaína } 0,125 \%\end{array}$ & Continuación Tabla 2 \\
\hline
\end{tabular}

Respecto al comportamiento de los signos vitales maternos durante el transoperatorio, en términos generales, las variables frecuencia cardiaca, presión arterial sistólica (PAS) y diastólica (PAD) y los niveles de saturación arterial de oxígeno, exhibieron un comportamiento similar al momento de la cirugía y la técnica de anestesia neuoraxial implementada. Se presentaron 21 casos de hipotensión sostenida (trece en la técnica epidural, cinco con anestesia combinada y tres con el abordaje espinal) y no se registraron casos de hipoxemia materna (Tabla 3). 


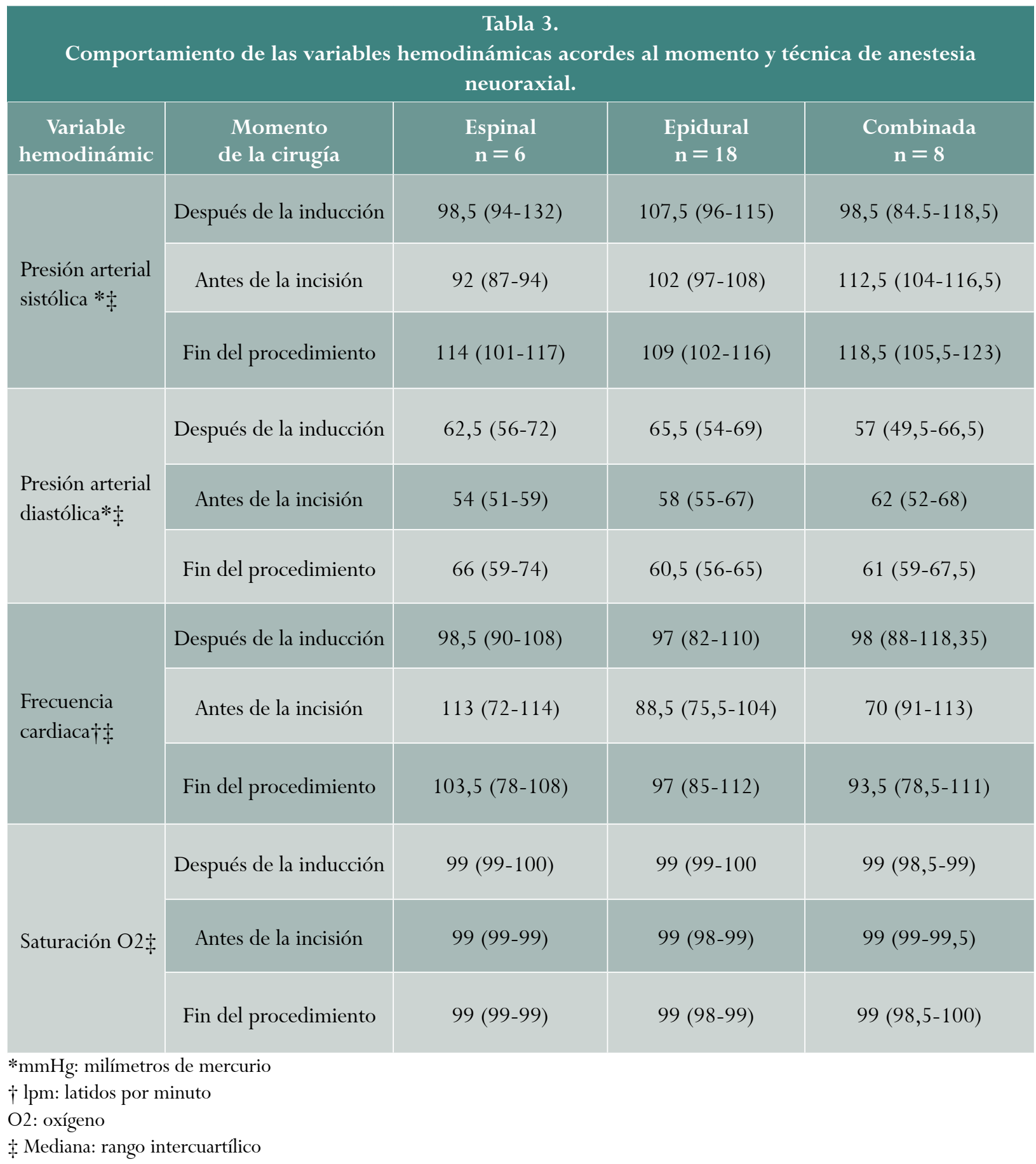

El 65,6\% de las participantes experimentaron parto pretérmino y el $18,7 \%$ ruptura prematura de membranas. Una gestante presentó un episodio de sangrado genital que se autolimitó en el posoperatorio inmediato sin que requiriera manejo específico. Se presentaron tres casos de edema pulmonar materno (dos en pacientes con técnica espinal y uno con anestesia combinada) y no se registraron casos de mortalidad materna o de lesión de víscera hueca asociados al procedimiento (Tabla 4). Se presentaron 16 muertes fetales (12 maternas perdieron un feto y 2 maternas perdieron ambos fetos) y nueve neonatales. De las 32 gestantes (64 fetos) atendidas en la institución con información completa al momento del alta, no fue factible obtener información del resultado perinatal para cuatro gestantes (pérdida al seguimiento) (Tabla 5 y 6). 


\begin{tabular}{|c|c|c|c|c|}
\hline Desen & lace materno segú & $\begin{array}{l}4 . \\
\text { tipo de } t\end{array}$ & euroaxial. & \\
\hline & Frecuencia olobal & Frec & egún la té & lestésica \\
\hline Nombre del desenlace & $\mathbf{n}=32$ & $\begin{array}{c}\text { Espinal } \\
n=6\end{array}$ & $\begin{array}{l}\text { Epidural } \\
\mathrm{n}=18\end{array}$ & $\begin{array}{c}\text { Combinada } \\
n=8\end{array}$ \\
\hline Parto pretérmino, n (\%) & $21(65,6)$ & $4(66,6)$ & $13(72,2)$ & $4(50,0)$ \\
\hline $\begin{array}{l}\text { Ruptura prematura de } \\
\text { membranas, n (\%) }\end{array}$ & $6(18,7)$ & $0(0,0)$ & $5(27,7)$ & $1(12,5)$ \\
\hline Hipotensión sostenida, n (\%) & $21(65,6)$ & $3(50,0)$ & $13(72,2)$ & $5(62,5)$ \\
\hline Edema pulmonar materno, n (\%) & $3(9,3)$ & $2(33,3)$ & $0(0,0)$ & $1(12,5)$ \\
\hline
\end{tabular}

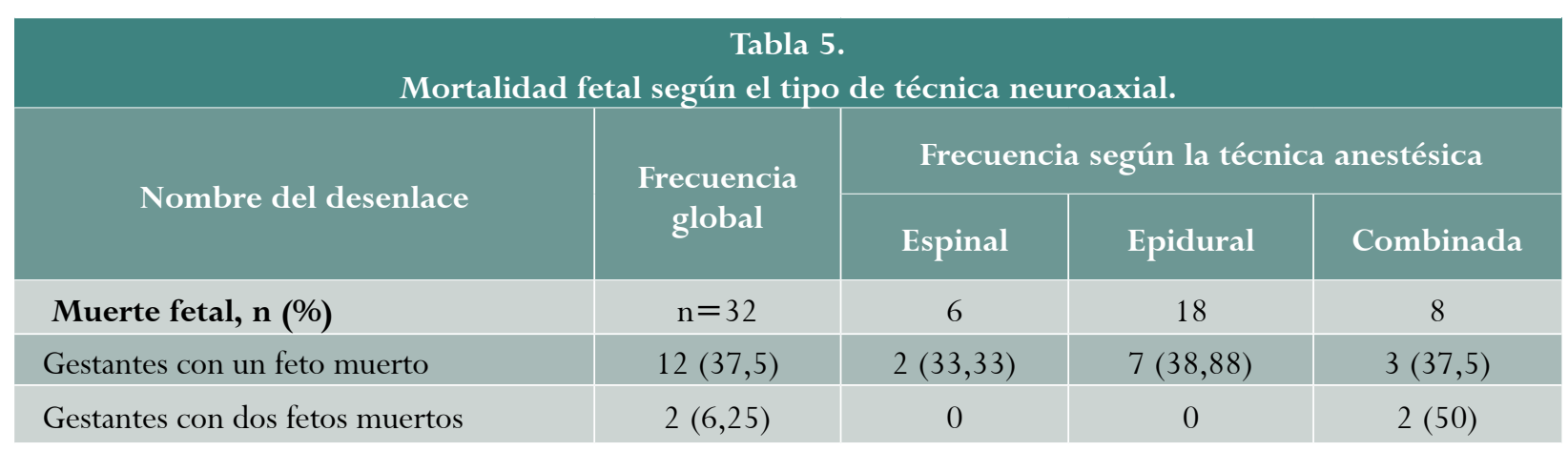

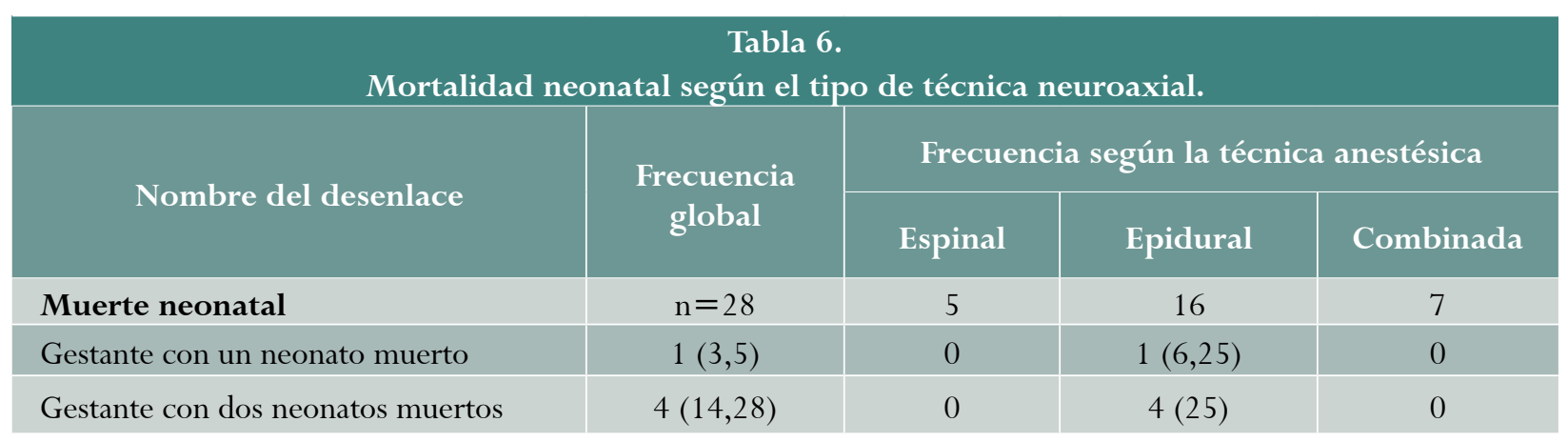

\section{DISCUSIÓN}

El STFF es una condición específica del embarazo gemelar monocorial (1) que incrementa la frecuencia de resultado perinatal adverso $(4,9)$ si no se brinda un tratamiento adecuado (6). En la actualidad, la evidencia es escasa e insuficiente con respecto al impacto que puede tener el uso de las diferentes técnicas de anestesia neuroaxial para el manejo de las gestantes con STFF programadas para fetoscopia y fotocoagulación láser $(1,2,17,21,23,27)$.
Este estudio de cohorte retrospectiva, con 32 participantes, analizó la frecuencia de complicaciones maternas, fetales y neonatales según el tipo de anestesia neuroaxial utilizado. La población estuvo constituida preponderantemente por mujeres jóvenes, multíparas, con sobrepeso, sin patología de base, quienes cursaban la segunda mitad de la gestación. En el 87,5\% de los casos la intervención se realizó de emergencia ante la evidencia de compromiso fetal; predominó el estadio II de Quintero, y se implementó el uso de anestesia 
epidural (56,25\%), seguido por la anestesia combinada (25\%), utilizando como terapia de mantenimiento remifentanil, fentanil o propofol.

Las características de la población objeto de estudio son similares a las reportadas en la literatura $(14,23)$. El diagnóstico de STFF puede pasar desapercibido a lo largo del primer trimestre de la gestación, en virtud del pobre desempeño que ofrecen los marcadores tempranos de compromiso fetal (28), aspecto que explica, en parte, por qué la mayoría de los casos se detectan durante el segundo trimestre y en estadios avanzados de la enfermedad (II y III de Quintero) (1), requiriendo la realización de un procedimiento de emergencia $(11,25)$.

En concordancia con lo documentado en otros estudios, la técnica neuroaxial representa el abordaje predilecto para este tipo de pacientes (2,14,17,21,23,29-31), dejando como alternativa para casos selectos el uso de la anestesia general con intubación orotraqueal $(14,17,21)$. El motivo para esta elección probablemente radica en que el uso de la técnica neuroaxial se encuentra vinculado con un menor compromiso en la presión de perfusión úteroplacentaria en comparación con la anestesia general (22). Si bien la superioridad de una técnica neuroaxial sobre otra no es clara, actualmente se considera que la técnica espinal quizás se asocia con una mayor frecuencia de bradicardia fetal (6 vs. $8 \%$ ), cuando se compara frente a otras técnicas neuroaxiales $(13,32)$. Este aspecto podría explicar el uso preferente de la técnica epidural sobre la espinal o combinada (18).

En lo que respecta a los resultados maternos, fetales y neonatales, este estudio documentó que el comportamiento de los signos vitales maternos exhibe un patrón similar entre las diferentes técnicas de anestesia neuroaxial. No obstante, llama la atención la presencia de 21 casos de hipotensión sostenida $(65,6 \%)$, trece de ellos con la técnica epidural (72,2\%). Aspecto que recuerda lo reportado en la literatura, en donde se describe la presencia de hipotensión sostenida entre el 7,4 y el 75\% para pacientes llevadas a cesárea con anestesia neuroaxial (33-35). Esta frecuencia esperada del evento tiene su origen en los cambios hemodinámicos maternos que genera este tipo de técnica anestésica (v. g. reducción del gasto cardiaco al modificar la resistencia vascular periférica) (33-35).

Por otro lado, resalta que el $65,6 \%$ de las participantes experimentaron parto pretérmino, 18,7\% ruptura prematura de membranas y el registro de tres casos de edema pulmonar. No se presentaron casos de mortalidad materna, pero se documentaron 16 muertes fetales $(25 \%)$ y 9 neonatales $(16,1 \%)$ entre los fetos que conformaron la cohorte. Si bien, el parto pretérmino y ruptura prematura de membranas son condiciones comunes durante el posoperatorio de esta población (68\% y $28 \%$, respectivamente) $(10,22)$, se registró una menor frecuencia con respecto a lo reportado en la literatura, probablemente como consecuencia del manejo rutinario con tocolíticos y de la vigilancia clínica estrecha.

En lo que respecta al desarrollo de edema pulmonar, es prudente recordar que las técnicas neuroaxiales generan bloqueo simpático, desencadenando episodios de hipotensión, los cuales requieren el uso de mayores volúmenes de líquidos intravenosos y de agentes vasopresores (36). Hecho que, sumado a los cambios fisiológicos del embarazo (v.g. hipervolemia materna toda vez que se trata de un embarazo múltiple) junto al uso de irrigación uterina para mejorar la visualización endoscópica (36) explican -en parte- la aparición de edema pulmonar en este tipo de pacientes. Finalmente, si bien la frecuencia de muerte fetal y neonatal fue alta en la cohorte (25\% y $16,1 \%$ respectivamente), los hallazgos registrados son similares a lo reportado en estudios previos, en donde se documenta sobrevidas menores al 75\% (24). La frecuencia de desenlaces adversos pueden ser atribuibles a que se trata de una condición que per se, amenaza la vida y el pronóstico de la gestación.

Este estudio tiene algunas limitaciones. Debido a la baja frecuencia de la enfermedad, sumado a que se trata de una intervención médica compleja, el tamaño de la cohorte para cada una de las técnicas anestésicas es reducido. Este aspecto conlleva la posibilidad de no haber podido documentar la ocurrencia de ciertos 
resultados adversos asociados a la intervención o a la técnica anestésica, dado el bajo poder (37). Otra posible falencia radica en la naturaleza retrospectiva de la información, aspecto que puede acarrear algunas imprecisiones en el registro de los datos (38). Finalmente, dado que no se implementó el uso técnicas efectivas para el control de la confusión, los resultados observados pueden deberse a la presencia de variables extrañas que pueden interferir con la asociación causal (38).

No obstante, este estudio también posee algunas fortalezas metodológicas. Dado que la entidad participante es centro de referencia para el suroccidente colombiano y que atiende pacientes pertenecientes al régimen contributivo y subsidiado, es probable que la población objeto de análisis sea representativa del área geográfica seleccionada. Por otra parte, en consideración del tipo de procedimiento y dadas las características de los desenlaces objeto de estudio, se tiene certeza de la exposición y de que los desenlaces de interés no se encontraban presentes al comienzo del periodo de observación. Finalmente, también sobresale el bajo número de pérdidas al seguimiento (12\%) y la duración óptima del periodo de observación para los integrantes que conformaron la cohorte (tres meses posnatales de seguimiento).

\section{CONCLUSIÓN}

En pacientes con STFF que requieren FL, el uso de la anestesia epidural, espinal o combinada, probablemente se asocia con un comportamiento similar al de las variables hemodinámicas maternas durante los momentos de la cirugía. Los profesionales que brindan atención a estas gestantes deben estar alerta ante la aparición de hipotensión sostenida, edema pulmonar, parto pretérmino, ruptura prematura de membranas y muerte fetal o neonatal. Se requieren estudios prospectivos que evalúen la seguridad y la efectividad de las diferentes técnicas de anestesia, idealmente bajo el contexto de un ensayo controlado aleatorizado.

\section{REFERENCIAS}

1. Rueda-Ruiz N. Síndrome de transfusión feto fetal. Medicas UIS. 2016;29(3):61-71. Disponible en: http://www.scielo.org.co/scielo.php?script $=$ sci arttext\&pid $=$ S0121-03192016000300061\&lng $=\mathrm{e}$

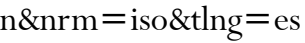

2. Yoda H. Fetal and neonatal circulatory disorders in twin to twin transfusion syndrome (The secondary publication). J Nippon Med Sch. 2019;86:192-200. https://doi.org/10.1272/jnms.JNMS.2019_86-301

3. Schwarz U, Galinkin JL. Anesthesia for fetal surgery. Semin Pediatr Surg. 2003;12(3):196-201. https://doi. org/10.1016/S1055-8586(03)00025-8

4. Simpson LL. Twin-twin transfusion syndrome. Am J Obstet Gynecol. 2013;208(1):3-18. https://doi. org/10.1016/j.ajog.2012.10.880

5. Molina-Giraldo S, Hincapié-Porras C. Cirugía fetal láser en síndrome de transfusión feto feto y restricción selectiva del crecimiento intrauterino. Rev Peru Ginecol y Obstet. 2018;64(4):587-97. https://doi. org/10.31403/rpgo.v64i2128

6. Gómez-Castro LA, Parra G, Vergara F, Parra M, Gómez M, Cuéllar R, et al. A case report and literature review in twin-twin transfusion syndrome stage III, fetoscopy - guided laser therapy, complicated with ballantyne syndrome and selective IUGR of fetus donor. Rev Colomb Obstet Ginecol. 2010;61(4):341-7. https://doi.org/10.18597/rcog.257

7. Habli M, Yen Lim F, Crombleholme T. Twin-to-twin transfusion syndrome: a comprehensive update. Clin Perinatol. 2009;36(2):391-416. https://doi. org/10.1016/j.clp.2009.03.003

8. Quintero RA, Morales WJ, Allen MH, Bornick PW, Johnson PK, Kruger M. Staging of twin-twin transfusion syndrome. J Perinatol. 1999;19(8):550-5. https://doi.org/10.1038/sj.jp.7200292

9. Bamberg $\mathrm{C}$, Hecher $\mathrm{K}$. Update on twin-to-twin transfusion syndrome. Best Prac Res: Clin Obstet Gynaecol. 2019; 58:55-65. https://doi.org/10.1016/j. bpobgyn.2018.12.011

10. Myers LB, Bulich LA, Hess P, Miller NM. Fetal endoscopic surgery: Indications and anaesthetic management. Best Pract Res Clin Anaesthesiol. 2004;18(2):231-58. https://doi.org/10.1016/j. bpa.2004.01.001 
11. Roberts D, Neilson JP, Kilby MD, Gates S. Interventions for the treatment of twin-twin transfusion syndrome, Cochrane Database of Systematic Reviews. 2014;30(1): CD002073. https://doi. org/10.1002/14651858.CD002073.pub3

12. Tran KM. Anesthesia for fetal surgery. Semin Fetal Neonatal Med. 2010;15(1):40-5. https://doi.org/10.1016/j. siny.2009.05.004

13. Littleford J. Effects on the fetus and newborn of maternal analgesia and anesthesia: A review. Can J Anesth. 2004;51(6):586-609. https://doi.org/10.1007/ BF03018403

14. Myers LB, Watcha MF. Epidural versus general anesthesia for twin-twin transfusion syndrome requiring fetal surgery. Fetal Diagn Ther. 2004;19(3):286-91. https:// doi.org/10.1159/000076713

15. Sviggum HP, Kodali BS. Maternal anesthesia for fetal surgery. Clin Perinatol. 2013;40(3):413-27. https://doi. org/10.1016/j.clp.2013.05.012

16. Buck F, Deprest J, Velde M. Anesthesia for fetal surgery. Curr Opin Anaesthesiol. 2008;21(3):293-7. https://doi. org/10.1097/ACO.0b013e3282fe6e70

17. Vasco Ramírez M. Anesthesia for fetal surgery. Colomb J Anesthesiol. 2012;40(4):268-72. https://doi. org/10.1016/j.rcae.2012.07.006

18. Tran KM, Chatterjee D. New trends in fetal anesthesia. Anesthesiol Clin. 2020;38:605-19. https://doi. org/10.1016/j.anclin.2020.05.006

19. Pardo MMR. Basics of Anesthesia. ClinicalKey. 2018. Disponible en: https://www.clinicalkey.es/\#!/browse/ book/3-s2.0-C20140048964

20. Noguchi S, Tanaka M, Terui K. The first national survey of anesthesia techniques for fetal therapies in Japan. J Anesth. 2019;33(6):665-9. https://doi.org/10.1007/ s00540-019-02690-w

21. Rossi AC, Kaufman MA, Bornick PW, Quintero RA. General vs local anesthesia for the percutaneous laser treatment of twin-twin transfusion syndrome. Am J Obstet Gynecol. 2008;199(2):137.e1-137.e7. https:// doi.org/10.1016/j.ajog.2007.12.008

22. Nivatpumin P, Pangthipampai P, Jirativanont T, DejArkom S, Triyasunant N, Tempeetikul T. Anesthetic techniques and incidence of complications in fetoscopic surgery. J Med Assoc Thail. 2016;99(5):60210. Disponible en: https://pubmed.ncbi.nlm.nih. gov/27501618/
23. Rustico MA, Lanna MM, Faiola S, Schena V, Dell'Avanzo M, Mantegazza V, et al. Fetal and maternal complications after selective fetoscopic laser surgery for twin-to-twin transfusion syndrome: A single-center experience. Fetal Diagn Ther. 2012;31(3):170-8. https://doi.org/10.1159/000336227

24. van de Velde M, van Schoubroeck D, Lewi LE, Marcus MAE, Jani JC, Missant C, et al. Remifentanil for fetal immobilization and maternal sedation during fetoscopic surgery: A randomized, double-blind comparison with diazepam. Anesth Analg. 2005;101(1):251-8. https://doi.org/10.1213/01.ANE.0000156566.62182. $\mathrm{AB}$

25. Organización Panamericana de la Salud (OPS). Clasificación estadística internacional de enfermedades y problemas relacionados con la salud (10 revisión). Washington, D.C.: OPS; 1995.

26. Ministerio de Salud y Protección Social. Resolución 2238 de 1 de diciembre de 2020. Disponible en: https:// consultorsalud.com/wp-content/uploads/2020/12/ Resolucion-No.-2238-de-2020-CUPS-2021.pdf

27. Shah AD, Border WL, Crombleholme TM, Michelfelder EC. Initial fetal cardiovascular profile score predicts recipient twin outcome in twin-twin transfusion syndrome. J Am Soc Echocardiogr. 2008;21(10):11058. https://doi.org/10.1016/j.echo.2008.05.004

28. Stagnati V, Zanardini C, Fichera A, Pagani G, Quintero RA, Bellocco R, et al. Early prediction of twin-to-twin transfusion syndrome: Systematic review and metaanalysis. Ultrasound Obstet Gynecol. 2017;49(5):57382. https://doi.org/10.1002/uog.15989

29. Kiefer N, Suter SF, Berg C, Gembruch U, Weber SU. Peridurale Anästhesie zur Fetoskopie: Retrospektive Analyse einer Einjahreskohorte. Anaesthesist. 2017;66(1):28-33. https://doi.org/10.1007/s00101 016-0253-5

30. Vasco M, Valencia G CM. Anesthesia for nonobstetric surgery in pregnancy. Clin Obstet Gynecol. 2020;63(2). https://doi.org/10.1097/GRF.0000000000000532

31. Ring LE, Ginosar Y. Anesthesia for fetal surgery and fetal procedures. Clinics in Perinatology. 2019; 46:80116. https://doi.org/10.1016/j.clp.2019.08.011

32. Mardirosoff C, Dumont L, Boulvain M, Tramèr MR. Fetal bradycardia due to intrathecal opioids for labour analgesia: A systematic review. BJOG An Int J Obstet Gynaecol. 2002;109(3):274-81. https://doi. org/10.1111/j.1471-0528.2002.01380.x 
33. Klöhr S, Roth R, Hofmann T, Rossaint R, Heesen M. Definitions of hypotension after spinal anaesthesia for caesarean section: Literature search and application to parturients. Acta Anaesthesiol Scand. 2010;54(8):90921. https://doi.org/10.1111/j.1399-6576.2010.02239.x

34. Ngamprasertwong P, Habli M, Boat A, Lim FY, Esslinger H, Ding L, et al. Maternal hypotension during fetoscopic surgery: Incidence and its impact on fetal survival outcomes. Sci World J. 2013;2013. https://doi. org/10.1155/2013/709059

35. Šklebar I, Bujas T, Habek D. Spinal anaesthesiainduced hypotension in obstetrics: Prevention and therapy. Acta Clin Croat. 2019;58(1):2019. https://doi. org/10.20471/acc.2019.58.s1.13

36. Duron VD, Watson-Smith D, Benzuly SE, Muratore CS, O'Brien BM, Carr SR, Luks FI. Maternal and fetal safety of fluid-restrictive general anesthesia for endoscopic fetal surgery in monochorionic twin gestations. J Clin Anesth. 2014;26(3):184-90. https://doi.org/10.1016/j. jclinane.2013.10.010

37. Andrade C. Sample size and its importance in research. Indian J Psychol Med. 2020;42(1):102. https://doi. org/10.4103/IJPSYM.IJPSYM_504_19

38. Kleinbaum, D. G., Kupper, L. L., Morgenstern H. Epidemiologic Research: Principles and Quantitative Methods. New York: Wiley; 1982.

\section{CONTRIBUCIÓN DE LOS} AUTORES

Luis Felipe Laverde-Martínez: concepción de la idea y diseño, recolección de la información, adquisición de los datos, análisis estadístico, planeación del artículo, escritura del artículo, revisión de contenido intelectual y aprobación final.

Laura Marcela Zamudio-Castilla: concepción de la idea, adquisición de los datos, análisis estadístico, planeación del artículo, escritura del artículo, revisión de contenido intelectual y aprobación final.

Akemi Arango-Sakamoto: planeación del artículo, análisis e interpretación de los datos, escritura del artículo, revisión de contenido intelectual y aprobación final.

Natalia Satizábal-Padridin: concepción de la idea, planeación del artículo, escritura del artículo, revisión de contenido intelectual y aprobación final.

Leidy Johanna López-Erazo: concepción de la idea, planeación del artículo, revisión de contenido intelectual y aprobación final.

Einar Sten Billefals-Vallejo: concepción de la idea, planeación del artículo, revisión de contenido intelectual y aprobación final.

Yuliana Angélica Orozco-Peláez: concepción de la idea, recolección de los datos, análisis y revisión de los datos, planeación del artículo final, revisión de contenido intelectual y aprobación final. 